\title{
Matthias Schöning
}

\section{»Zäsur«. Probleme einer historiographischen Angewohnheit}

\section{Mehr als nur ein Ereignis}

Den Begriff »Zäsur« zu gebrauchen, ist für Zeitdiagnostiker und Historiker offensichtlich gleichermaßen reizvoll. ${ }^{1}$ Das gilt insbesondere dann, wenn man den Ersten Weltkrieg untersucht, und bestätigt sich, wenn vom Zweiten Weltkrieg bis hin $\mathrm{zu}$ 9/11 die Gewaltgeschichte des 20. und beginnenden 21. Jahrhunderts zum Thema wird. Doch auch in alltäglichen Zusammenhängen wird gerne auf den Begriff "Zäsur " oder sein vorrangiges Synonym »Einschnitt « ${ }^{2}$ zurückgegriffen, um einen qualitativen Unterschied zwischen zwei zeitlich getrennten Zuständen zu bezeichnen. Wer »Zäsur « sagt, nominiert ein Ereignis als historisches Datum, das zwei substantiell unterschiedliche Zeiten trennt. ${ }^{3}$

Die historische "Zäsur" ist insofern ein Sonderfall des Ereignisses. Jede "Zäsur" setzt ein realgeschichtliches Ereignis voraus, das durch die Bestimmung als "Zäsur» qualifiziert wird. Umgekehrt ist jedoch nicht jedes Ereignis eine "Zäsur«. Schreibt man dem Ereignis die konstitutiven Merkmale Nicht-Alltäglichkeit, Diskontinuität und Bedeutsamkeit $\mathrm{zu},{ }^{4}$ so hebt sich die »Zäsur« durch

1 Trotzdem »hat der Begriff der Zäsur gegenüber dem der Epoche und der Periodisierung eine nur vergleichsweise geringe theoretische Betrachtung erfahren« (Martin Sabrow: Deutungszäsur und Erfahrungszäsur. In: Ders.: Zeitgeschichte schreiben. Von der Verständigung über die Vergangenheit in der Gegenwart. Göttingen: Wallstein 2014, S. 160-177, hier S. 162).

2 Vgl. z. B. Günter Rohrmoser: Zäsur. Wandel des Bewußtseins. Stuttgart: Seewald 1980, S. 11: »Zäsur - Wandel des Bewußtseinsı: der Titel meint einen Einschnitt, in diesem Fall einen Wandel der Epoche. Man ist gezwungen innezuhalten. Geschichtliche Erinnerung ist unerläßlich, wenn man den Andrang dessen bestehen will, was neu und anders ist."

3 Besonders hilfreich für die Überprüfung des allgemeinen Wortgebrauchs ist die Nutzung des Digitalen Wörterbuchs der deutschen Sprache der Berlin-Brandenburgischen Akademie der Wissenschaften, das nicht nur verschiedene Wörterbücher auswertet, sondern auch ein grammatisch strukturiertes Wortprofil anbietet, das u. a. die typischen Attribute von »Zäsur» vor Augen führt. Abrufbar unter http://www.dwds.de/?qu=Zäsur (Stand: 26. 8. 2014).

4 Vgl. z. B. Hans Robert Jauß: Versuch einer Ehrenrettung des Ereignisbegriffs. In: Reinhart Koselleck u. Wolf-Dieter Stempel (Hg.): Geschichte - Ereignis und Erzählung. München: Fink 1973, S. 554-560, hier S. 555. 
eine spezifische Steigerung aller drei Dimensionen hervor. Als Konsequenz fordert sie eine grundlegende Umwertung der bis dato geltenden NormalitätsAnnahmen.

Indem das als "Zäsur« qualifizierte Ereignis Zeiten trennt, konstituiert es zugleich Epochen - oder, um genau zu sein, es konstituiert den Endpunkt einer älteren und den Anfangspunkt einer jüngeren Epoche. Goethes Kommentar zur Kanonade von Valmy - „Von hier und heute geht eine neue Epoche der Weltgeschichte aus, und ihr könnt sagen, ihr seid dabei gewesen « - ist dafür typisch. ${ }^{5}$ Als Ereignis ist die "Zäsur« jedoch zugleich ein Sonderfall der sogenannten `Epochenschwelle`, dem historiographischen Standardausdruck für die Bezeichnung von Übergangsphasen zwischen Epochen. ${ }^{6}$ Sie teilt mit dieser die Funktion der Trennung zweier grundlegend verschiedener Zeitphasen. Die Form der Trennung ist jedoch eine andere. Dem langsamen, gleitenden Übergang über die metaphorische `Schwelle`, die selbst eine räumliche, bzw. zeitliche Ausdehnung hat, steht die Plötzlichkeit gegenüber, die mit dem Begriff »Zäsur» betont wird. Mit einem Mal ist alles anders.

\section{Die »Zäsur « in zeitdiagnostischem Gebrauch}

Die Implikation, dass eine paradigmatische Revision `des Weltbildes` nötig sei, und zwar ohne Zeitverzug, unter dem bewegenden Eindruck des unerwarteten Ereignisses, dürfte die Anziehungskraft des »Zäsur«-Konzepts unter den Zeitgenossen eines entsprechenden Ereignisses ausmachen. Wer den Begriff zeitdiagnostisch gebraucht, verschafft sich - imaginär oder tatsächlich - einen Zeitvorsprung gegenüber den Adressaten seiner Rede. Er hat bereits erkannt, dass es umzudenken gilt, und kann verlangen, dass man ihm folgt. Schnelligkeit generiert Autorität.

In markanter Weise zur Anwendung gekommen ist dieses »Zäsur«-Konzept zuletzt nach 9/11. Während die Bush-Administration sofort Pläne für ihren ıwar on terror`schmiedet, rufen Intellektuelle das Ende der Postmoderne aus. ${ }^{7} \mathrm{Ge}-$

5 Er wird freilich erst in Goethes autobiographischem Text, „Campagne in Frankreich«, knapp dreißig Jahre nach der Nacht im Feldlager formuliert. Vgl. Nicolas Boyle: Goethe. Der Dichter in seiner Zeit. Bd. 2. München: C. H. Beck 1999, S. 167.

6 Zur Begriffsgeschichte von Epoche mit ihrer charakteristischen Bedeutungsverschiebung von der Bezeichnung eines Zeitpunkts zur Bezeichnung eines Zeitabschnitts vgl. Manfred Riedel: Epoche, Epochenbewußtsein. In: Joachim Ritter (Hg.): Historisches Wörterbuch der Philosophie. Bd. 2. Darmstadt: WBG 1972, Sp. 596-599.

7 Besonders scharfkantig ausgefallen sind im deutschen Sprachraum die Einlassungen des Wahlamerikaners Hans Ulrich Gumbrecht im Merkur: In eine Zukunft gestoßen. Nach dem 11. September 2001. In: Merkur 55 (2001) H. 631, S. 1048-1054. Ähnlich wie der amerikanische Publizist Roger Rosenblatt im Time Magazine stellt er die Diagnose, dass das `Zeitalter 
naue Beobachter haben anhand der Verortung der jeweiligen "Zäsur« sogar verschiedene Phasen des Diskurses über den Terroranschlag zu unterscheiden versucht, die allesamt aus der Diagnose einer "Zäsur» die Ermächtigung zu Veränderungen ableiten, während für die Zeithistoriker zunächst einmal in Frage steht, ob überhaupt eine Zäsur vorliegt. ${ }^{8}$

Urszenen der appellativen "Zäsur«-Diagnostik sind die intellektuelle Mobilmachung bei Beginn des Ersten Weltkriegs und die Kämpfe um dessen Deutung in der Zeit der Weimarer Republik. Für 1914 ist zum Beispiel an Thomas Mann zu denken, der seine "Gedanken im Kriege« im Novemberheft der Neuen Rundschau äußert. Mann begrüßt den Krieg nicht als Fortsetzung der 1871 begonnenen Geschichte des deutschen Kaiserreiches unter preußischer Führung, sondern als Umkehrpunkt. Die Epoche der Prosperität soll mit einem Krieg enden, der die Rückkehr zum Wesentlichen der Nation als zugleich deren weltgeschichtlicher Mission bringt. Die Prüfung der Nation und jedes Einzelnen werden daher ausdrücklich begrüßt. Unter den Schicksalsschlägen, so die implizierte Trivialpsychologie, fällt alles Äußerliche ab, so dass der Wesenskern der deutschen Nation freigelegt wird. Kern der Zeitdiagnose ist das Postulat einer kulturellen "Zäsur", die so konstruiert ist, dass sie das aktuelle Zeitgeschehen und das persönliche Profil des Autors im literarischen Feld einander akkommodieren. Der Überraschung seines Umfelds zum Trotz bleibt Thomas Mann sich in gewisser Hinsicht treu: Weil der Autor die Hinfälligkeit der Welt, die 1914 einstürzt, seit seinen Buddenbrooks fortwährend beleuchtet hat, versteht er sich

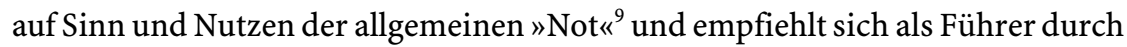
die Katastrophe.

der Ironie an sein Ende gekommen sei. Die Zielscheibe von Rosenblatt und Gumbrecht ist offensichtlich das, was mit dem Schlagwort Postmoderne bezeichnet wird, mit der man wiederum einen nicht nur ästhetischen, sondern auch ethischen Relativismus assoziiert. Vgl. Michael C. Frank: 9/11 als Zäsur. Zur Karriere eines Topos in Politik, Medien und akademischem Diskurs. In: Stephan Packard u. Ursula Hennigfeld (Hg.): Abschied von 9/11? Distanznahmen zur Katastrophe. Berlin: Frank \& Timme 2013, S. 15-34, hier insb. S. 18, der zur Frage der "Zäsur « urteilt, 9/11 sei nicht "an und für sich schon eine Wende « gewesen, sondern "wurde mit großem Aufwand zu einer solchen gemacht» (ebd., S. 16).

$8 \mathrm{Zu}$ den verschiedenen »Zäsur«-Setzungen vgl. ebd., S. 15f. Zur grundsätzlichen Infragestellung, dass es sich um eine »Zäsur« handele vgl. Michael Butter, Birte Christ u. Patrick Keller (Hg.): 9/11. Kein Tag, der die Welt veränderte. Paderborn u. a.: Schöningh 2011, S. 7-12, hier S. $7 \mathrm{f}$.: »Im Unterschied zu Studien, die fälschlicherweise darauf beharren, dass der 11. September eine Zäsur darstellt, relativiert dieses Buch die Bedeutung dieses Ereignisses." Vgl. auch Thorsten Schüller u. Sascha Seiler (Hg.): Von Zäsuren und Ereignissen. Historische Einschnitte und ihre mediale Verarbeitung. Bielefeld: transcript 2010.

9 Thomas Mann: Gedanken im Kriege [1914]. In: Ders.: Große kommentierte Frankfurter Ausgabe. Werke - Briefe - Tagebücher. Bd. 15.1: Essays II: 1914-1926. Hg. u. textkr. durchges. von Hermann Kurzke. Frankfurt/M.: Fischer 2002, S. 27-46, hier S. 34. 


\section{Auch die »Zäsur« kann eine Geschichte haben}

Es ist allerdings weder Zufall, noch verdankt es sich dem `Genie` des Autors, dass Thomas Mann bereits kurze Zeit nach Beginn des Ersten Weltkriegs den Sinn des Krieges als Wendemarke bestimmt. Vielmehr liegen die Deutungsmuster schon lange bereit: "Der Erste Weltkrieg überraschte die Zeitgenossen zwar in vielerlei Hinsicht: in seiner Dauer, seiner Grausamkeit und Hartnäckigkeit und vor allem in seiner demoralisierenden Wirkung auf die beteiligten Völker. Aber selbst noch in diesen unerwarteten Erscheinungsformen erfüllte sich eine Erwartungsstruktur, die zum Teil schon seit langem angelegt war. ${ }^{10}$ Im letzten Quartal des 19. Jahrhunderts mehren sich nicht nur in Deutschland die Stimmen, die einen größeren Krieg als den zuletzt zwischen Deutschland und Frankreich ausgetragenen kommen sehen. Von der politischen Zeitdiagnostik bis zu Zukunftsromanen werden Szenarien eines künftigen Krieges durchgespielt und selbst die anti-militaristische Prognostik rechnet mit der Gefahr eines einschneidenden Krieges. ${ }^{11}$

Dem erstrebten Neubeginn korrespondieren von Beginn an Abbruch von Entwicklungen und Umwertung von Werten. Dass dieser Vorgang schmerzhaft ausfallen kann, wird keineswegs ausgeschlossen - im Gegenteil. Einem sich selbst der Schwäche verdächtigenden Zeitalter ${ }^{12}$ beglaubigt der Schmerz vielmehr die Echtheit des Einschnitts. Es ist deshalb keineswegs bloß eine spontane Hypertrophie, wenn Thomas Mann ausdrücklich von einer »Utopie des Unglücks« spricht. ${ }^{13}$

Noch zehn Jahre nach dem Krieg verfolgt ein anderer Zögling aus der großen Gruppe emphatischer Nietzsche-Leser, Ernst Jünger, dieselbe Strategie und plädiert für Fortsetzung des Zerstörungswerks als Voraussetzung des nächsten, nunmehr rechten` Neuanfangs. ${ }^{14}$ Nach dem Ende des Krieges müssen viele Hoffnungen begraben werden, doch das Schema wiederholt sich. So wie Heinrich Mann seinem jüngeren Bruder nur mit einer alternativen »Zäsur«, nicht

10 Lucian Hölscher: Die Entdeckung der Zukunft. Frankfurt/M.: Fischer 1999, S. 200.

11 Vgl. ebd., S. $202 \mathrm{ff}$.

12 Vgl. Joachim Radkau: Das Zeitalter der Nervosität. Deutschland zwischen Bismarck und Hitler. München, Wien: Hanser 1998, insb. S. 295-309 u. 389-407.

13 Mann: Gedanken (Anm. 9), S. 33 (Hervorh. M.Sch.).

14 Vgl. Ernst Jünger: Das Abenteuerliche Herz. Erste Fassung: Aufzeichnungen bei Tag und Nacht. Stuttgart: Klett Cotta 1987, S. 95: "Man muß erkennen, [...] daß wieder wie im 15. Jahrhundert der Rauch der Scheiterhaufen über der Landschaft steht. [...] Die Beschäftigung des Deutschen zu dieser Zeit ist die, von allen Ecken der Welt Material herbeizuschleppen, um den Brand zu nähren, den er unter seinen Begriffen gestiftet hat. So ist es denn kein Wunder, daß alles, was brennbar ist, in vollen Flammen steht. [...] [D]as Schreckliche dieses Vorgangs, der sich im menschlichen Bestande vollzieht, [...] hat nichts Problematisches, sondern im Gegenteil etwas sehr Notwendiges". 
aber mit einer Zurückweisung des Denkmusters entgegentreten konnte, ${ }^{15}$ so antwortet auch Walter Benjamin auf Ernst Jünger nur mit einer Umcodierung. Der Diskurs ist stark. Es geht nur darum, in seinem Rahmen das am meisten bewegende Bild zu finden, um die Diagnose der "Zäsur« auf die eigene Seite zu ziehen. Walter Benjamin: "Eine Generation, die noch mit der Pferdebahn zur Schule gefahren war, stand unter freiem Himmel in einer Landschaft, in der nichts unverändert geblieben war als die Wolken und in der Mitte, in einem Kraftfeld zerstörender Ströme und Explosionen, der winzige gebrechliche Menschenkörper. ${ }^{16}{ }^{1}$ - Man sieht: Als Deutungsmuster für Ereignisse hat auch die »Zäsur« eine Geschichte.

\section{Belegstellen für den historiographischen Begriffsgebrauch}

Der Eindruck, den der skizzierte Gebrauch des Konzepts »Zäsur« hinterlässt, schürt zweierlei Verdacht. Der Begriff »Zäsur« und seine Synonyme scheinen immer dann herangezogen zu werden, wenn es gilt, die Differenz zwischen einem Vorher und einem Nachher zu markieren und als besonders hervorzu-

15 Heinrich Mann antwortet genau ein Jahr später, im November 1915 in Die weißen Blätter, einerseits mit einer geschickten Inversion aller von seinem Bruder ins Feld geführten Begriffe und auch von dessen rhetorischer Strategie. Andererseits jedoch deutet auch er den Krieg als Zäsur. Der aufdringlichen Pose des Bekenners, der aus der Stimmung der Situation weltgeschichtliche Prognosen ableitet und sich ebenso sehr zum praeceptor germaniae aufschwingt wie er dem Begehren nach symbolischer Verschmelzung mit dem Volkskörper nachgibt, setzt Heinrich Mann in seinem Zola-Essay von 1915 die Haltung des Vermittlers entgegen, der nicht über sich, sondern über einen anderen spricht und Geschichtsziele und Utopien nicht für Einzelvölker reklamiert. Trotz der geschickten Inversion bleibt ein Denkmuster jedoch intakt. Auch Heinrich Mann erwartet vom Krieg einen einschneidenden Wandel, einen Einschnitt in die deutsche Geschichte. Zugespitzt könnte man sagen, dass wir es mit einem spiegelverkehrten Denken zu tun haben, das entlang der Achse einer tiefgreifenden Zäsurerwartung, die beide Texte gleichermaßen bestimmt, gespiegelt, bzw. Punkt für Punkt umcodiert wird. Der Krieg ist auch für diesen Intellektuellen eine Produktivkraft des gesellschaftlichen Fortschritts. Es werden nur andere Geschichtsziele ausgegeben. Oder noch weniger: Es werden nur farblich anders gestaltete Ziele proklamiert, die gemeinsam haben, dass nach dem Krieg eine stärker integrierte Gesellschaft stehen soll, sei es als Mitbestimmungsdemokratie, sei es als vorpolitische Gemeinschaft, die sich unmittelbar konstituiert. Die Metaphorik ist dann sogar nahezu identisch, wenn es heißt: »Reiche, die Schranken aufrichten vor dem Glück ihrer Völker, Reiche, die unter Panzern die Menschenliebe ersticken, verderbte und gewalttätige Reiche, sie mögen hinsinken, sie geben den besten Dünger für die Saat einer verjüngten Menschheit. [...] Über den Tod hinweg gehen [sie] in das verjüngte Leben hinein, das Demokratie heißt (Heinrich Mann: Zola [1915]. In: Ders.: Essays und Publizistik. Bd. 2: Oktober 1904 bis Oktober 1918. Hg. von Manfred Hahn. Bielefeld: Aisthesis 2012, S. 148-209, hier S. 179).

16 Walter Benjamin: Erfahrung und Armut [1933]. In: Ders.: Illuminationen. Ausgewählte Schriften. Bd. 1. Hg. von Siegfried Unseld. Frankfurt/M.: Suhrkamp 1977, S. 291-296, hier S. 291. 
heben, ohne sie genauer zu bestimmen. Die Verführungskraft erwächst eher aus dem strategischen Vorsprung, einen Bedarf als erster anzuzeigen, als dem Vermögen, diesen auch zu decken. Wer sich verspätet, dem bleibt die Möglichkeit, mit einer besonders dringlichen Version die nötigen Anhänger zu finden. In jedem Fall dominiert die appellative Funktion klar über den Erklärungswert des Konzepts »Zäsur«. Wie verhält sich zu diesem objektsprachlichen Gebrauch nun die Historiographie?

Eine kurze Recherche zum Wortgebrauch im Bereich der Geschichtsschreibung bestätigt zunächst die enorme Rekurrenz dieser Begrifflichkeit, vor allem wenn man weitere gleichsinnig verwendete Begriffe wie $» Z e i t e n b r u c h ~ «{ }^{17}$ und Umschreibungen mit einbezieht. So schreibt etwa Barbara Tuchman - mit The Guns of August. The Outbreak of World War I bzw. ihrem in der deutschen Fassung meist nur August 1914 betitelten Buch die Verfasserin eines populären Klassikers über den Ersten Weltkrieg - in ihrem anschließenden Werk, das sich mit der Vorgeschichte befasst: »Der Große Krieg von 1914 bis 1918 hat sich wie ein breiter Streifen verbrannter Erde zwischen uns und die Zeit davor geschoben. $\aleph^{18}$ Tuchman liefert ein Bild für das, was eine »Zäsur" typischerweise charakterisiert. Eine mit Tod assoziierte Linie trennt zwei Lebensformen voneinander, deren jüngere sich von der älteren durch einen nicht wieder gut zu machenden Verlust unterscheidet. Die existentielle Aufladung folgt dem Topos, den bereits Teilnehmer und Zeitgenossen am Ersten Weltkrieg konstituiert haben.

Ein ähnliches Pathos der Bilder findet man auch im Umkreis von 9/11. Typisch für die historiographische Literatur ist dagegen weniger, das Konzept neuerlich aufzuladen, als vielmehr dessen Existentialität stillschweigend zu beerben. Der eigene Forschungsgegenstand hat ein größeres Gewicht, wenn er nicht nur als Ereignis, sondern als "Zäsur« angesprochen wird. Explizit von "Zäsur« sprechen in der jüngsten Literatur zum Ersten Weltkrieg u. a. Benjamin Ziemann und Ulrich Sieg. ${ }^{19}$ Ebenso starke Kandidaten für entsprechende $\mathrm{Zu}$ -

17 Vgl. Inka Mülder-Bach (Hg.): Modernität und Trauma. Beiträge zum Zeitenbruch des Ersten Weltkriegs. Wien: WUV 2000. Vgl. auch Jörn Leonhard: Die Büchse der Pandora. Geschichte des Ersten Weltkriegs. München: C. H. Beck 2014, S. 11: „Der tiefe Bruch wurde zum prägenden Merkmal - ob in stilisierten Kindheitserfahrungen oder als Orientierungspunkt einer Generation, die nach 1918 die Konsequenzen des Krieges erfuhr.»

18 Barbara W. Tuchman: Der stolze Turm. Ein Porträt der Welt vor dem Ersten Weltkrieg 1890-1914. München u. a.: Knaur 1969, S. 11.

19 Vgl. Benjamin Ziemann: Gewalt im Ersten Weltkrieg. Töten - Überleben - Verweigern. Essen: Klartext 2013, S. 8:»[...] Frage [...], ob der Schock und die traumatische Zäsur des Weltkriegs zur Konstituierung und Formierung von Subjektivität beitrugen und in welchen symbolischen Formen sich diese Suche nach einem mit dem Krieg kompatiblen Subjekt vollzog.» Ulrich Sieg: Geist und Gewalt. Deutsche Philosophen zwischen Kaiserreich und Nationalsozialismus. München: Hanser 2013, S. 126: „Gleichwohl markiert [die sogenannte 
schreibungen sind die oft als "Zivilisationsbruch» bezeichnete Shoa $(\mathrm{h})^{20}$ und das Jahr 1945, das gerne auch als »Stunde Null« betitelt wurde. ${ }^{21}$ Weniger gewaltsam als vergleichsweise friedlich waren dagegen die demokratischen Revolutionen in Osteuropa 1989. ${ }^{22}$ Beispiele für den Gebrauch des »Zäsur «-Begriffs angesichts historischer Geschehnisse von geringerer Reichweite finden sich z. B. bei Aleida Assmann, in eigens den "Zäsuren« der deutschen Geschichte gewidmeten Sammelbänden, einem Lehrbuch zur Geschichte der Außenpolitik der Bundesrepublik Deutschland 1949-1990 und sogar im Titel einer Interpretation der Bundestagswahlen des Jahres 2005..$^{23}$ - Das letzte Beispiel zeigt, wie gering

Judenzählung des deutschen Heeres 1916] eine Zäsur in der deutschen Geschichte«. Prominent auch Michael Stolleis: Der lange Abschied vom 19. Jahrhundert. Die Zäsur von 1914 aus rechtshistorischer Perspektive. Berlin, New York: de Gruyter 1997. Zäsuren in der nichtwissenschaftlichen Sachbuchliteratur zum Ersten Weltkrieg auf der Spur ist Jens Wörner: Geist - Macht - Krieg. Perspektiven auf die Moderne in ihrer fremden Vertrautheit. In: Christian Meierhofer, Michael Schikowski u. ders. (Hg.): Materialschlacht. Der Erste Weltkrieg im Sachbuch. Non Fiktion 8/9 (2013/14) H. 2/1, S. 147-177.

20 Dan Diner (Hg.): Zivilisationsbruch. Denken nach Auschwitz. Frankfurt/M.: Fischer 1988. Vgl. auch Jürgen Habermas: Eine Art Schadensabwicklung. Kleine politische Schriften VI. Frankfurt/M.: Suhrkamp 1987, darin insb. die Aufsätze "Geschichtsbewußtsein und posttraditionale Identität. Die Westorientierung der Bundesrepublik« und »Vom öffentlichen Gebrauch der Historie. Das offizielle Selbstverständnis der Bundesrepublik bricht auf", S. 159-179 u. 243-255. Vgl. auch Amir Eshel: Zeit der Zäsur. Jüdische Dichter im Angesicht der Shoah. Heidelberg: Winter 1999 und Ashraf Noor (Hg.): Erfahrung und Zäsur. Denkfiguren der deutsch-jüdischen Moderne. Freiburg/Br.: Rombach 1999.

21 Im Unterschied zu den bisher genannten Ereigniskomplexen Erster Weltkrieg und Shoa/ Holocaust, die nach wie vor als »Zäsuren« bezeichnet werden, hat sich bezüglich des Jahres 1945 die Überzeugung durchgesetzt, dass eine Betitelung als "Stunde Null« angesichts starker Kontinuitäten im Bereich der administrativen Eliten, aber auch in der Kunst und Literatur allzu irreführend ist. Vgl. Bernd Hüppauf: Einleitung: Schwierigkeiten mit der Nachkriegszeit. In: Ders. (Hg.): "Die Mühen der Ebenen«. Kontinuität und Wandel in der deutschen Literatur und Gesellschaft 1945-1949. Heidelberg: Winter 1981, S. 7-20 und Peter Davies, Stephen Parker u. Matthew Philpotts: The Modern Restoration. Re-thinking German Literary History 1930-1960. Berlin, New York: de Gruyter 2004.

22 Vgl. Bernd Florath (Hg.): Das Revolutionsjahr 1989. Die demokratische Revolution in Osteuropa als transnationale Zäsur. Göttingen: Vandenhoeck \& Ruprecht 2001.

23 Vgl. Aleida Assmann: Geschichte im Gedächtnis. Von der individuellen Erfahrung zur öffentlichen Inszenierung. München: C. H. Beck 2007, S. $53 \mathrm{f}$.: »Nicht jeder Generation ist es beschieden, ihren geschichtlichen Auftritt und Abtritt als eine historische Zäsur zu markieren. Die 68er Generation unterscheidet sich von allen anderen Generationen des 20. Jahrhunderts dadurch, dass ihre Identität nicht an historische Vorgaben geknüpft ist (wie den ersten oder zweiten Weltkrieg), sondern dass sie selbst eine historische Zäsur setzte. 1968 wurde nicht erst nachträglich zu einer Zäsur, diese wurde auch von innen heraus durch eine Bewegung forciert - nicht nur in Deutschland, sondern gesamteuropäisch und transkontinental. Der epochale Bruch wurde erlebnisnah auf Teach-ins, Straßendemonstrationen und Popkonzerten vollzogen und war begleitet von einem Diskurs, in dem man (vielfach im Schulterschluss mit den 45ern) das Ende des Bestehenden und Geltenden deklamierte«; Alexander Gallus (Hg.): Deutsche Zäsuren. Systemwechsel seit 1806. Köln, Weimar, Wien: Böhlau 2006; Martin Broszat (Hg.): Zäsuren nach 1945: Essays zur Peri- 
die Halbwertzeit der Empfindung, einem historischen "Einschnitt« beizuwohnen, sein kann. Was vor zehn Jahren als »Zäsur« betitelt werden konnte, ist heute kaum mehr als einschneidend rekonstruierbar. ${ }^{24}$

\section{Etymologie}

Der dokumentierte Verbreitungsgrad des historischen Konzepts ${ }^{25}$ "Zäsur« und die daraus abzuleitende Tendenz zur schrankenlosen Verwendung macht seinen Erklärungswert zweifelhaft und erneuert die Frage nach den Gründen für den häufigen Gebrauch.

Zunächst ist an die Begriffsgeschichte zu erinnern. Zum festen Sprachgebrauch gehört der Ausdruck »Zäsur « erst in der Musiktheorie ${ }^{26}$ und dann in der Verslehre, wo er "Einschnitte« entweder »im Inneren eines Versfußes ${ }^{27}$ oder heute gebräuchlicher - nach einer festgelegten Silbenzahl, die ein Metrum konstituiert, bezeichnet. ${ }^{28}$ Im spezielleren Fall der Deklamation von Lyrik wer-

odisierung der deutschen Nachkriegsgeschichte. München: Oldenbourg 1990; Ulrich Lappenküper: Die Außenpolitik der Bundesrepublik Deutschland 1949-1990. München: Oldenbourg 2008, S. 27: "Die Wahl Willy Brands zum vierten Bundeskanzler am 21. Oktober 1969 markiert in der Geschichte der Bundesrepublik Deutschland eine tiefe historische Zäsur. Erstmals seit ihrem Bestehen leite ein Sozialdemokrat die Regierungsgeschäfte«; Frank Decker: Die Zäsur. Konsequenzen der Bundestagswahl 2005 für die Entwicklung des deutschen Parteiensystems. In: Berliner Republik. Das Debattenmagazin 6 (2005). Abrufbar unter http://www.b-republik.de/b-republik.php/cat/8/aid/928/title/Die_Zaesur (Stand: 22.8.2014).

24 Der Name von Angela Merkel, die in Konsequenz dieser Wahl zum ersten Mal zur deutschen Bundeskanzlerin gewählt wurde, fällt in dem Artikel von Frank Decker nicht! Zur Zeit- und Standpunktabhängigkeit vgl. auch Sabrow: Deutungszäsur (Anm. 1), S. 167: »Zäsuren sind [...] perspektivenabhängig, wie sich nicht nur zwischen den verschiedenen nationalen Meistererzählungen zeigt, sondern mehr noch zwischen Mit- und Nachwelt. Besonders im Medienzeitalter und der mit ihm verbundenen kommunikativen Verdichtung werden sie oft ausgerufen und schnell wieder vergessen, wie es etwa der Jahrhundert- und Jahrtausendzäsur erging, die von einem starken Bewusstsein der Zeitenwende begleitet wurde und rückblickend ihren Zäsurencharakter rasch wieder eingebüßt hat. Nicht selten werden zunächst dramatisch erscheinende Einschnitte durch den wachsenden Abstand wieder eingeebnet«. Sabrows Aufsatz verdanke ich auch die Hinweise auf Lappenküper und Decker (Anm. 23).

25 Ich spreche im Weiteren vom »Konzept `Zäsur « Begriffs »Zäsur« und seiner Synonyme und Hyponyme zu bezeichnen - im Unterschied z. B. zum auch in der Lyrikanalyse verwendeten Begriff "Zäsur".

26 Vgl. den Artikel »Caesura« im Zedlerschen Universallexikon, Bd. 5, Sp. 107. Abrufbar unter http://www.zedler-lexikon.de (Stand: 26.8.2014).

27 Im Unterschied zur »Diärese«: Kristin Felsner, Holger Helbig u. Therese Manz: Arbeitsbuch Lyrik. Berlin: Oldenbourg 2009, S. 48.

28 Vgl. zum Verhältnis Diärese/Zäsur auch Jost Schneider: Art. »Zäsur«. In: Klaus Weimar 
den "Sprechpausen oft generell als Zäsuren bezeichnet.«" ${ }^{29}$ Der Duden online nennt explizit den alltäglichen, wenngleich »bildungssprachlich[en]« Wortgebrauch zur Bezeichnung eines »Einschnitt[s] (besonders in einer geschichtlichen Entwicklung)«. Als Synonyme kennt der Duden: »Bruch, Einschnitt, markanter Punkt, Unterbrechung «. ${ }^{30}$ Das Digitale Wörterbuch der Deutschen Sprache (DWDS) betont den "scharfe[n], einschneidende[n] « Charakter der "politische[n], historische[n], weltgeschichtliche[n] [...] Zäsur «. ${ }^{31}$ Dazu passt die Wortherkunft. »Zäsur« stammt von lateinisch caesūra ab, eigentlich »das Hauen, Fällen, Einschneiden«, abgeleitet vom Verb caedere »(um-, nieder) schlagen, hauen, (ein-, auf-, heraus)schneiden «, ${ }^{32}$ dessen Bedeutungsspektrum gemäß der einschlägigen Wörterbücher des Lateinischen damit allerdings noch keineswegs erschöpft ist.

\section{Nachhaltigkeit und Veränderungsintensität}

Die semantische Aura des Gewaltsamen lässt das Konzept »Zäsur« besonders angemessen dafür erscheinen, solche geschichtlichen Ereignisse zu bezeichnen, bei denen das (Über-)Maß der angewendeten Gewalt den wesentlichen qualitativen Unterschied zwischen Vorher und Nachher ausmacht und damit zugleich den Interpretationsbedarf hinsichtlich grundlegender Annahmen über die Natur des Menschen und die Grundlagen des Zusammenlebens hervorruft. Wenn es angesichts des Ereignisses auf Schnelligkeit ankommt, dann ist nun, im (zeit-)historischen Nachgang, Beharrlichkeit gefordert. Weil sich die historische Bedeutung der »Zäsur" nicht so leicht abgelten lässt, muss man immer wieder interpretatorisch zu ihr zurückkehren. Dabei kommt es nicht zuletzt darauf an, die vergangenen Schrecken in Erinnerung zu behalten und gerade nicht zu normalisieren. Eine "Zäsur« bleibt das historische Ereignis in der historiographischen Perspektive nur, wenn es in der Erinnerung weiter schmerzt, wenn es als >Wunde` fungiert, die sich nicht gänzlich schließt. ${ }^{33}$

(Hg.): Reallexikon der deutschen Literaturwissenschaft. Bd. 3. 3., neubearb. Aufl. Berlin, New York: de Gruyter 2003, Sp. 869 f., hier Sp. 869.

29 Felsner, Helbig u. Manz: Arbeitsbuch Lyrik (Anm. 27), S. 49.

30 Duden online. Abrufbar unter http://www.duden.de/rechtschreibung/Zaesur (Stand: 28.8. 2014).

31 Digitales Wörterbuch der deutschen Sprache (Anm. 3).

32 Ebd. Vgl. auch Friedrich Kluge: Etymologisches Wörterbuch der deutschen Sprache. 23. Aufl. Bearb. von Elmar Sebold. Berlin, New York: de Gruyter 1999, S. 904. Keinen Artikel "Zäsur« enthalten das Grimmsche Wörterbuch und das Historische Wörterbuch der Philosophie.

33 Vgl. Aleida Assmann: Erinnerungsräume. Formen und Wandlungen des kulturellen Gedächtnisses. München: C. H. Beck 1999, S. $241 \mathrm{ff}$. 
Angesichts der dominierenden Verknüpfung des "Zäsur«-Konzepts mit den Schrecken der Geschichte des 20. Jahrhunderts wirkt es daher unangemessen, wenn für ephemere Veränderungen wie Fernsehformate der Begriff der "Zäsur» bemüht wird. ${ }^{34}$ Will man sich trotzdem nicht auf >maßlose Gewaltanwendung als Kriterium für Ereignisse, die als »Zäsur« bezeichnet werden können sollen, festlegen, so könnte alternativ z.B. folgender Substitutionstest vorgenommen werden: Alle Ereignisse, die sich - metaphorischer Gebrauch eingeschlossen als Revolutionen bezeichnen lassen, weil sie plötzlich und veränderungsintensiv in das Leben vieler Menschen eingreifen und deren Alltag gleichsam >umkrempeln`, kommen zugleich als Kandidaten für historische "Zäsuren" in Frage. ${ }^{35}$ Dann wären z.B. "mediengeschichtliche Zäsuren « denkbar, ${ }^{36}$ die Veränderungen bezeichnen, die das kulturelle Leben srevolutionieren`, während einzelne Veränderungen innerhalb eines kulturellen Feldes, eines Mediums oder gesellschaftlichen Teilsystems, seien sie auch noch so markant, nicht als »Zäsur « in Frage kämen. Das wäre vereinbar mit der eingangs vorgenommenen Bestimmung von "Zäsuren" als Untergruppe von Ereignissen, die einen transgressiven Charakter in dem Sinne haben, dass sie mehrere Lebensbereiche gleichermaßen stark auf den Kopf stellen und ein entsprechend fundamentales Umdenken erfordern.

\section{Geschichte im Singular}

Trotzdem sollte man an die Synchronisierung der Veränderungen in den von einer "Zäsur" betroffenen Teilbereichen nicht zu große Erwartungen haben. Nimmt man z. B. den Ersten Weltkrieg, so lassen sich die Jahre 1914, 1916, 1917 und 1918 gleichermaßen gut als >eigentliche « Zäsur" nominieren, je nachdem ob man die Geschichte der Arbeiterklasse, die Geschichte des Antisemitismus in Deutschland, die Geschichte Amerikas oder die Verfassungsgeschichte verschiedener europäischer Staaten ins Zentrum stellt. Auch gesellschaftsgeschichtlich steckt der Teufel im Detail. Was macht man, wenn das groß angelegte, auf Befriedigung eines womöglich grundlegenden »Zäsurbedarfs $\aleph^{37}$ abzielende

34 Vgl. Joachim Michael: Telenovelas und kulturelle Zäsur. Intermediale Gattungspassagen in Lateinamerika. Bielefeld: transcript 2010.

35 Wenig distinktiv ist dagegen der Eingrenzungsversuch von Broszat: Zäsuren (Anm. 23), S. 10: „Die Frage nach Zäsuren der Geschichte geht [...] davon aus, daß nicht jedes Jahr der Geschichte gleich zu Gott ist, sondern daß es dicht beschriebene, aber auch ziemlich leere Blätter der Geschichte gibt«.

36 Vgl. Georg Christoph Tholen: Die Zäsur der Medien. Kulturphilosophische Konturen. Frankfurt/M.: Suhrkamp 2002.

37 Einen solchen diagnostiziert Odo Marquard: Temporale Positionalität. Zum geschichtlichen 
Konzept sich mit zunehmender Beobachtungsgenauigkeit sukzessive verflüchtigt?

Man kann zwar nachträglich an als "Zäsur« bestimmten Ereignissen wie dem Ersten Weltkrieg Differenzierungen vornehmen, wenn sich herausstellt, dass »die Zäsuren der Wirtschafts- und Sozialgeschichte und ebenso der Kulturgeschichte [...] anderen Logiken und Rhythmen des Wandels « folgen. ${ }^{38}$ Sicher lassen sich als »Zäsur» bereits etablierte Ereignisse derart auffächern. Fraglich ist jedoch, ob es Sinn hat, nach der Erkenntnis der Unverträglichkeit des "Zäsur«-Konzepts mit der historiographischen Differenzierung von Teilgeschichten am liebgewonnenen Begriff festzuhalten und ihn für allerhand historische Kleinphänomene und Spezialgeschichten freizugeben. Trotz der tatsächlichen Ubiquität des Begriffsgebrauchs sperrt sich der Begriff seinem Sinn nach gegen allzu freigiebige Pluralisierung von Geschichte in Geschichten. Vielmehr scheint dem historiographischen Konzept "Zäsur« eine umgekehrte Richtung eigen zu sein, die es weniger für immer kleinere als vielmehr für die Generierung immer größerer Orientierungsmarken der europäischen oder sogar Globalgeschichte disponiert. ${ }^{39}$ Das Konzept "Zäsur « tendiert damit zu einer `Geschichte im Singular` und impliziert einen Rest Geschichtsphilosophie. Insofern fragt es sich, inwiefern es überhaupt die Geschehnisse selbst sind, die den Gebrauch des Konzepts »Zäsur« bestimmen.

Zäsurbedarf des modernen Menschen. In: Reinhard Herzog u. Reinhart Koselleck (Hg.): Epochenschwelle und Epochenbewußtsein. München: Fink 1987, S. 343-352.

38 Sabrow: Deutungszäsur (Anm. 1), S. 166. Vgl. auch Stolleis: Abschied (Anm. 19), S. 5: „Die [...] These vom >langen Abschied، [vom 19. Jh.] lautet: Zäsuren sind auf Plausibilität angelegte Merkzeichen der Historiographie. Es sind Hilfskonstruktionen zur Bewältigung von Datenmengen. Um ihre Ungleichzeitigkeit geht es in den folgenden sechs Abschnitten. Denn Rechtsgeschichte, Sozial- und Wirtschaftsgeschichte, Verfassungsgeschichte und politische Geschichte datieren den Abschied vom 19. Jahrhundert unterschiedlich." Vgl. weiter Michael Prinz u. Matthias Frese: Sozialer Wandel und politische Zäsuren seit der Zwischenkriegszeit. Methodische Probleme und Ergebnisse. In: Dies. (Hg.): Politische Zäsuren und gesellschaftlicher Wandel im 20. Jahrhundert. Paderborn: Schöningh 1996, S. 1-31.

39 Von der Möglichkeit weltgeschichtlicher Zäsuren geht eine Ringvorlesung der Universität Hildesheim aus. Abrufbar unter http://www.uni-hildesheim.de/neuigkeiten/weltgeschicht liche-zaesuren-in-gesellschaft-und-politik-1989-2001-2011 (Stand: 31.8.2014). Vgl. auch Angela Siebold: 1989 - eine Zäsur von globaler Reichweite? In: Aus Politik und Zeitgeschichte vom 10.6.2014, Nr. 24-26: Aufbruch '89, S. 3-9. Für gesamteuropäische Zäsuren vgl. Florath: Revolutionsjahr (Anm. 22) und Andreas Thier, Guido Pfeifer u. Philipp Grzimek (Hg.): Kontinuitäten und Zäsuren in der Europäischen Rechtsgeschichte. Frankfurt/M. u. a.: Lang 1999. 


\section{Affinität von »Zäsur« und Gewalt}

Zieht man die für die Pragmatik des "Zäsur«-Konzepts initialen Ereignisse heran, dann fällt allen Vorbehalten gegenüber Gewaltereignissen als Epochengrenzen zum Trotz die Affinität von »Zäsur« und Gewalt auf. Insbesondere bei Bezugnahme auf den Ersten Weltkrieg und die Shoa ist es das als irrational, ja als verstörend unverständlich qualifizierte Verhältnis zwischen der von großen geschichtlich konstituierten, staatlich organisierten und insofern srationalen Gruppen aufgewendeten Gewalt und deren Nutzen, der die Rede vom »Zeiten-« oder "Zivilisationsbruch" motiviert. Im Falle des Ersten Weltkriegs sind es vor allem die in das Jahr 1916 fallenden Schlachten an der Westfront, namentlich der deutsche Angriff auf Verdun und die britisch-französische Somme-Offensive, nach deren jeweiligem Scheitern zu vernachlässigende Geländegewinne exorbitanten Opferzahlen gegenüberstehen. Im Fall der Shoa ist es die zweckspezifische Rationalität der zunehmend systematischen Vernichtung der europäischen Juden, die der Unbegreiflichkeit des Zwecks selbst gegenübersteht. In beiden Fällen ist es die Form der Gewalt, die die nachträglichen Interpreten vor Probleme stellt. Weil es sich nicht um quasi-natürliche Gewaltausbrüche, nicht um spontane, situative `Eruptionen ` von Hass und Tötungslust handelt, sondern um Ereignisse, die ein ausgesprochen hohes Maß an langfristiger Planung und arbeitsteiliger Koordination voraussetzen (Qualitäten also, die in anderen $\mathrm{Zu}$ sammenhängen als systemische Rationalitäten beschrieben werden und Gesellschaften als modern kennzeichnen), irritieren sie die Beobachter. Während Lynchjustiz und Pogrom noch als historische Rückstände innerhalb moderner Gesellschaften beschrieben werden können, die darauf verweisen, dass die Ausbreitung der als Zivilisationsprozess begriffenen Moderne schrittweise und lückenhaft vonstattengeht, setzen die beiden Weltkriege und die nationalsozialistische Judenvernichtung die spezifischen Kräfte und Potentiale moderner Gesellschaften gerade voraus.

\section{Differenz von Erwartung und Erfahrung}

Trotzdem ist es nicht die Form der Gewalt allein, die den Begriffsgebrauch reguliert. Die Geschichte der Moderne ist offensichtlich nicht zugleich eine Zivilisationsgeschichte oder der Zivilisationsprozess ist nicht als Prozess des Rückgangs, sondern nur als Formwandel der Gewalt zu beschreiben. ${ }^{40}$ Wer aber

40 Vgl. zum Komplex Moderne, Zivilisationsprozess und Gewalt Peter Imbusch: Moderne und Gewalt. Zivilisationstheoretische Perspektiven auf das 20. Jahrhundert. Wiesbaden: VS Verlag für Sozialwissenschaften 2005. 
erkennt, dass Modernisierung nicht zugleich Pazifizierung ist, muss seine Geschichtsvorstellung revidieren, denn sie könnte bis dato allzu optimistisch gewesen sein. Um diese Umschrift nun wiederum zu rechtfertigen, attribuiert der Interpret dem Ereignis den einschneidenden Charakter, den eigentlich erst die dem Ereignis gegenüber notwendigerweise späteren - und zum Teil reichlich verspäteten ${ }^{41}$ - Neuinterpretationen zum Ausdruck bringen.

Untersucht man also die Ursache der Irritation, die die Gewaltanwendung bei den Beobachtern hervor- und den "Zäsur «-Begriff auf den Plan ruft, genauer, so wird man auf deren Erwartungshorizont verwiesen. Nicht das Ereignis allein macht den Unterschied, sondern das Verhältnis zwischen dem, was sich ereignet, und dem, was im weltanschaulich gerahmten Erwartungshorizont als erwartbar gegolten hat. ${ }^{42}$ Das Zusammenspiel von Erwartung und Erfahrung eint zeitgenössische und historiographische Perspektiven. Sieht man von marxistisch inspirierten Geschichtsphilosophien ab, die sich selbst freilich nicht als philosophisch, sondern als wissenschaftlich bezeichnen würden, dann dürfte sich schnell Einigkeit darüber herstellen lassen, dass historische "Zäsuren" historiographische Kategorien sind und nicht objektive 'Einschnitter in der Geschichte selbst. Allein das Merkmal der Singularität der Shoa bzw. des Holocaust, das im Historikerstreit eine zentrale Rolle gespielt und die Hauptparteien unversöhnlich hat gegenüberstehen lassen, ${ }^{43}$ stellt eine Ausnahme dar. Die Konsequenz daraus ist in gewissem Sinne paradox: Weil die Tatsache der Judenvernichtung selbst die »Zäsur « ist (und nicht die Differenz zwischen Geschehen und Erwartungshorizont), beherrscht das Problem der Repräsentation (und ihrer Grenzen) die akademischen Diskussionen und die Debatten kreisen um die Frage, »welche formalen Erfordernisse für die verantwortungsvolle Darstellung des Holocaust erfüllt sein müssen. $\aleph^{44}$ Auch das größte Beharren darauf, dass die

41 Vergleicht man die Deutungsgeschichte der Judenvernichtung mit der intellektuellen Wahrnehmung des Ersten Weltkriegs, dann kann man die öffentliche Diskussion des Holocaust bzw. der Shoa als 'verspätet` bezeichnen, ohne zu vergessen, dass bereits die öffentliche Diskussion über den Ersten Weltkrieg in der Breite erst zehn Jahre nach Kriegsende Fahrt aufnimmt.

42 Vgl. den klassischen Aufsatz von Reinhart Koselleck: `Erfahrungsraum` und `Erwartungshorizont - zwei historische Kategorien [1976]. In: Ders.: Vergangene Zukunft. Zur Semantik geschichtlicher Zeiten. Frankfurt/M.: Suhrkamp 1989, S. 349-375.

43 Vgl. u. a. Ernst Nolte: Zwischengeschichtslegende und Revisionismus? sowie ders.: Vergangenheit, die nicht vergehen will. In: Rudolf Augstein u. a.: »Historikerstreit«. Die Dokumentation der Kontroverse um die Einzigartigkeit der nationalsozialistischen Judenvernichtung. München: Piper 1987 S. 13-35 u. 39-47. Vgl. dagegen Jürgen Habermas: Eine Art Schadensabwicklung. Die apologetischen Tendenzen in der deutschen Zeitgeschichtsschreibung. In: Ebd., S. 62-76 und z. B. Jürgen Kocka: Hitler sollte nicht durch Stalin und Pol Pot verdrängt werden. In: Ebd., S. 132-141.

44 Frank R. Ankersmit: Die postmoderne Privatisierung der Vergangenheit. In: Herta Nagl- 
Geschehnisse selbst und nicht erst deren Darstellung - d. h. je nach Konzeption: Erinnerung, Bezeugung - die Bedeutung des Ereignisses konstituiert, zieht eine metahistorische Diskussion nach sich, die notwendigerweise von der historischen auf die historiographische Ebene führt - und darüber hinaus.

\section{Eine limitierte Sprechmöglichkeit}

Die methodologische Überzeugung, es in Konzepten wie der »Zäsur« mit historiographischen >Anschauungsformen $\mathrm{zu}$ tun $\mathrm{zu}$ haben, die sich durch wissenspragmatische Funktionalität rechtfertigen, erübrigt nicht die Frage nach der je spezifischen Beschaffenheit des Verhältnisses zwischen Begriff und Sache. Zieht man zum Vergleich den Begriff »Epoche " heran, mit dem ein Zeitabschnitt der Geschichte als in je spezifischer Hinsicht temporär selbstidentisch bezeichnet wird, dann erscheint beim Gebrauch des Begriffs "Zäsur« die performative Seite stärker ausgeprägt. Bei Verwendung des »Zäsur«-Begriffs dominiert die Sozialdimension über die Sachdimension. Wer »Zäsur!« sagt, bezieht sich stärker auf das Welt- und Geschichtsbild seiner Adressaten als auf das Geschehen, das seinem Appell als Anlass dient.

Die eine Epoche ist anders als die andere, "Romantik" etwas anderes als »Realismus«. Objektive Unterschiede dieser Art muss jede Geschichtsschreibung, die mit Epochen arbeitet, als grundsätzlich möglich voraussetzen. Nach "Zäsuren" dagegen ist die Welt nicht im gleichen >objektiven` Sinne anders wie nach dem Eintritt in eine neue "Epoche». Sie ist anders anders. Vor die normalgeschichtlichen Unterschiede, die der Wandel und die Fortschritte im Laufe der Jahre mit sich bringen, hat sich ein einziger gewaltiger Unterschied geschoben. Die Setzung einer "Zäsur» konstruiert eine andere Alterität als es Epochen tun. Sie holt eine Gewalt zurück in die Historiographie, die historisch spezifische Formen kennt, aber vielleicht gar nicht durch und durch geschichtlich ist.

Als historiographischer Terminus ist der Begriff »Zäsur« jedenfalls weitgehend leer. Er zeigt den Bedarf an Bestimmung eines eklatanten Unterschieds an, kann diesen aber nicht selbst befriedigen. Deskription ist nicht seine Domäne. Wo er gebraucht wird, schwingt vielmehr eine moralische Dringlichkeit mit, die den Begriff "Zäsur« kommunikativ riskant oder eben reizvoll macht. Wird die Dringlichkeit allgemein empfunden, erscheint der Begriffsgebrauch angemessen. Ist das nicht der Fall, kann der Verdacht entstehen, dass die eigene Sache brisanter erscheinen soll als sie ist. In beiden Fällen hat der Gebrauch eine

Docekal (Hg.): Der Sinn des Historischen. Geschichtsphilosophische Debatten. Frankfurt/ M.: Fischer 1996, S. 201-234, hier S. 216. 
symptomatische Qualität, die es nahe legt, ihn weniger zu verwenden als sein Vorkommen zu untersuchen.

Die Rede von der "Zäsur» stellt insofern eine limitierte, aber notwendige Sprechmöglichkeit dar, die besondere Situationen markiert. Einige Merkmale lassen sich aus dem Sprachgebrauch rekonstruieren. Anwendungsregeln zu formulieren, ist sinnlos. Zu bedenken bleibt nur, dass es auch als vergesslich erscheinen kann, jeden neuen Akt der Unmenschlichkeit zur »Zäsur« zu erklären. 